\title{
Marlene Freitas da Silva (1937-2005)
}

\author{
William A. RODRIGUES ${ }^{1}$
}

Marlene Freitas da Silva, cujo nome de solteira era Marlene Alves Freitas, nasceu em Manaus em 12 de agosto de 1937 e faleceu em sua terra natal, em 18 de outubro de 2005 em conseqüência de uma infecção generalizada após uma cirurgia de vesícula. Foi casada com o bancário Cassiano Silva, já falecido, com quem teve duas filhas: Simone e Cassilene. Dedicou quase 50 anos de suas vida aos estudos botânicos, em particular sobre a taxonomia das Leguminosas, de cujos estudos deu valiosas contribuições para a ciência. Começou sua carreira em 1955 , no Instituto Nacional de Pesquisas da Amazônia - INPA, como aluna do Centro de Pesquisas Geomorfológicas Aplicadas na Amazônia e participante de uma expedição científica organizada pelo INPA ao Território de Rio Branco (hoje Roraima), sob a chefia do professor francês Francis Ruellan, quando ainda cursava o segundo grau do Colégio Estadual do Amazonas. Com o término do curso e extinção do Centro de Pesquisas Geomorfológicas, ela e seu colega de curso, Byron Wilson Pereira de Albuquerque (falecido em 2003), também futuro pesquisador do INPA, em 1956 foram lotados na Botânica, inicialmente como auxiliares de laboratório. Sua ascensão como pesquisadora na instituição foi alcançada paulatinamente, graças aos seus próprios méritos, competência, perspicácia, humildade, abnegação, coragem, seriedade, muito estudo, dedicação ao trabalho e autodeterminação. Em 1962, durante a gestão do Dr. Djalma Batista no INPA, foi-lhe oferecida uma bolsa de estudo para fazer o curso superior fora do Estado, porém como recém-casada, ausentar-se de Manaus por muito tempo era obviamente muito difícil. Preferiu aguardar por melhores oportunidades e continuar desfrutando de uma Bolsa de Iniciação Científica, concedida pelo INPA, para um treinamento na própria instituição, sobre Botânica Geral e Taxonomia Vegetal sob nossa orientação. Em 1960, participou no Rio de Janeiro de um Curso Básico de Botânica, patrocinado pelo Jardim Botânico do Rio de Janeiro e ministrado pela competente botânica, Dr ${ }^{\text {a }}$. Graziela Barroso. Em 1967, com a criação na Universidade Federal do Amazonas da especialização em Farmácia e Bioquímica, conseguiu por fim realizar os seus sonhos ao ingressar nesse curso e graduar-se em 1970. Em seguida, com a criação pelo INPA / FUA do primeiro curso de Pós-Graduação em Botânica, em Manaus, em 1973, ingressou nele e obteve o título de Mestre em 1976 com a dissertação intitulada "Revisão taxonômica do gênero Peltogyne Vogel (Leguminosae - Caesalpinioideae)" sob a orientação do Dr. Ghillean T. Prance. Em 1980, doutorou-se, também no INPA, com a tese intitulada "Revisão taxonômica do gênero Dimorphandra Schott (Leguminosae - Caesalpinioideae)". Publicou só ou em colaboração ao todo 89 trabalhos em diversas áreas do conhecimento da Botânica, sendo 13 livros , 8 capítulos de livros e 66 artigos diversos publicados em periódicos . Além disso, teve 86 artigos editados em forma de resumo em diversos eventos científicos. Nesses trabalhos demonstrou sua competência e os rasgos de sua personalidade e denodo científico e humano. Até aposentar-se em 1991 como pesquisadora do INPA, ocupou cargos importantes nessa instituição, entre eles o de chefe da Coordenadoria de Pesquisas em Botânica (CPBO) e o de curador do Herbário. Ministrou cursos na Pós-Graduação do INPA, orientou ou co-orientou 12 mestrandos, 4 doutorandos, 15 graduandos e 12 estudantes de Iniciação Científica. Apesar de aposentada, continuou até quase o fim de sua vida pesquisando e ministrando cursos na Pós-Graduação do INPA, orientando pós-graduandos, participando de vários projetos de pesquisa na Amazônia, foi chefe da divisão do Curso de Pós-Graduação em Botânica, conselheiro e chefe substituto do Conselho do Curso de PósGraduação em Botânica e membro do conselho editorial da revista Acta Amazonica e da revista Acta Botanica Brasilica, editada pela Sociedade Botânica do Brasil, no período de 1990 a 1994. Em 1983, a Sociedade Botânica do Brasil a incumbiu, sem consultá-la previamente, de organizar e presidir em Manaus o XXXV Congresso Nacional de Botânica, que acabou ocorrendo com muito sucesso no Hotel Tropical de Manaus, em janeiro do ano seguinte e por fim editorar em 1990 os Anais desse evento. No período de 1992 a 1994, fez parte do Conselho Superior da Sociedade Botânica do Brasil e o presidiu em 1984. Em 1983, ingressou no Instituto Tecnológico da Amazônia (UTAM) como professora titular do curso de Engenharia Florestal e entre 1993 a 1995 chegou a exercer a função de Diretora do Centro de Ensino e Pesquisas Florestais dessa mesma instituição de ensino. A partir de 2003, ingressou na Universidade Estadual do Amazonas (UEA) como Professora titular, ministrou cursos sobre morfologia e taxonomia e participou ativamente de projetos de pesquisa e orientação de pós-graduandos. A partir de 2003, passou a exercer o cargo de Coordenadora do Curso de Pós-Graduação da mesma instituição. Sua morte prematura, no auge de sua carreira e produtividade científica, foi muito sentida por todos os seus amigos e admiradores que a estimavam muito e usufruíam de seus vastos conhecimentos, em especial sobre a flora regional. Em vida, recebeu várias homenagens, entre eles a da Comenda do Mérito Farmacêutico em 2005, Membro de Honra da Academia Nacional Paulista de Farmácia em 1979, Placa INPA 50 Anos pelos relevantes serviços prestados para o desenvolvimento da Ciência e Tecnologia na Amazônia, em 2004 e outros mais, entre elas a consagração maior de ter o seu

${ }^{1}$ Departamento de Botânica - UFPR 
nome eternamente ligado a várias plantas novas. Escreveu também vários táxons novos, dentre eles a espécie Dimorphandra jorgei M. F. Silva, espécie nova esta dedicada ao seu extremado pai, Jorge de Deus Freitas, porque, segundo ela própria justificou no protólogo, "quando em vida, foi um exemplo de amor, honradez, dedicação à família e ao trabalho". Deixou lamentavelmente um grande vazio nas pesquisas regionais, já tão enormemente carente de competentes cientistas do nível de conhecimento que ela possuía no campo da botânica amazônica. Foi uma perda irreparável para a Amazônia e a Ciência, e, em particular, para aqueles que se orgulhavam de desfrutar de sua amizade e ensinamentos. Era incansável estudiosa e adorava a sua terra natal. Dedicou quase toda a sua vida à pesquisa e ao ensino regionais. Só se afastou de Manaus uma vez para completar seus estudos por 3 meses noJardim Botânico de Nova Iorque e uma outra para completar sua formação ainda incipiente em taxonomia vegetal, no Jardim Botânico do Rio de Janeiro. Possuía uma grande personalidade, era uma mãe exemplar, afável, comunicativa, alegre, generosa, corajosa e ao mesmo tempo muito exigente nas pesquisas dos que com ela trabalhavam ou recebiam orientação. Ela entrou na história do INPA como uma das pioneiras nos estudos botânicos da instituição. Por isso a sua foto no quadro de honra afixado no herbário do INPA não é mais que uma singela e merecida homenagem a ela pelo muito que fez em prol do engrandecimento da instituição, como um órgão mundialmente conhecido pelas suas pesquisas no âmbito da biota amazônica. Devemos realçar, também, sua dedicação ao ensino na pós-graduação do INPA e na UTAM e depois UEA, onde soube cativar seus discípulos e despertar vocações, sendo muito admirada e respeitada pela maioria de seus discípulos, vários deles hoje ilustres botânicos.

\section{TÁXONS NOVOS OU RECOMBINADOS POR MARLENE FREITAS DA SILVA E CO-AUTORES}

Styrax squamulosa M. F. Silva.Acta Amazonica 1 (1).: 23.1971 e 1.c. 1 (3): 23.1971

Styrax discolor M. F. Silva.Acta Amazonica 2 (1): $17 . .1972$

Styrax cinerea M. F, Silva. Acta Amazonica 1 (1): 23. 1971 el.c. 1 (3): 21.1971.

Sterculia duckei Eliz L. Taylor ex J. A. C. Silva \& M. F. Silva. Acta Amazonica 31 (3):375, f. 1-2. 2001.

Rauia prancei W.A. Rodrigues \& M. F. Silva.Acta Amazonica 1 (1): 21. 1971, f. 1.;Acta Amazonica 1 (2): 34. 1971.

Enterolobium barnebianum Mesquita \& M. F. Silva. Acta Amazonica. Supl. 14 (1/2): 153. 1984.

Caryocarglabrum subsp.parviflorum (A. C. Smith) G. T. Prance \& M. F. Silva. Fl. Neotropica. Monogr. 12: 43. 1973.

Caryocar glabrum subsp. album Prance \& M. F. Silva. Fl. Neotropica, Monogr.. 12: 43. 1973.

Caryocar brasiliense subsp. intermedium (Wittm.) Prance \& M. F. Silva. Fl. Neotropica, Monogr. 12: 26. 1973.

Peltogyne venosa subsp. densiflora (Benth.) M. F. Silva. Acta
Amazonica 6 (1) Supl. 50.1976

Peltogyne prancei M. F. Silva. Acta Amazonica 6 (1) Supl.: 44. 1976

Peltogyne parvifolia var. glabra M. F. Silva. Acta Amazonica 6 (1) Supl.: 43. 1976.

Peltogyne beterophylla M. F. Silva. Acta Amazonica 6 (1). Supl.: 30 (-33). 1976.

Peltogyne catingae subsp. glabra (W. A. Rodrigues) M. F. Silva. Acta Amazonica 6 (1). Supl. 24. 1976.

Peltogyne campestris var. rígida (Ducke) M. F. Silva. Acta Amazonica 6 (1) Supl.: 21. 1976.

Peltogyne paniculata subsp. pubescens (Benth.) M. F. Silva. ActaAmazonica 6 (1); Supl.: 6 (1): 38.1976.

Dimorphandra williamii M. F. Silva. Acta Amazonica 11: 57. 1981.

Dimorphandra macrostachya subsp. glabrifolia (Ducke) M. F. Silva. Fl. Neotropica. Monogr. 44: 106. 1986.

Dimorphandra macrostachya subsp. congestiflora (Sprague \& Sandwich) M. F. Silva.Fl. Neotropica. Monogr. 44: 109. 1986.

Dimorphandra loretensis M. F. Silva. Acta Amazonica 11: 54.1981

Dimorphandra jorgei M. F. Silva. Acta Amazonica 11: 53. 1981.

Dimorphandra cuprea subsp. velutina (Ducke) M. F. Silva.Fl. Neotropica. Monogr. 44: 97. 1986.

Dimorphandra cuprea subsp. ferruginea (Ducke) M. F. Silva. Fl. Neotropica. Monogr. 44: 94. 1986.

\section{TÁXONS NOVOS DEDICADOS ATÉ AGORA À MARLENE FREITAS DA SILVA}

Connarus marleneae Forero ("marlenei"), 1980

Cynometra marleneae A. S. Tavares, (1990) 1991

Talisia mollis var. marleneana G. Guarim Neto, 1983

Couepia marleneae Prance ("marlenei"), 1974

Licania marleneae Prance ("marlenei"), 1976

Virola marleneae W.A. Rodrigues ("marlenei"), 1977

Heteropterys marleneae W. R. Anderson, 1982

\section{LIVROS PUBLICADOS OU ORGANIZADOS POR MARLENE FREITAS DA SILVA}

LOUREIRO, A. A.; SILVA, M. F. 1968. Catálogo de madeiras da Amazônia. Belém, Pará. SUDAM. 2v. 433 p.

PRANCE, G. T.; SILVA, M. F. 1973. A monograph of Caryocaraceae. Flora Neotropica. New York, OFN. v. 12. 75p.

PRANCE, G. T.; SILVA, M. F. 1975. Árvores de Manaus. INPA, Manaus, AM, 312 p. ilustr.

SILVA, M. F. 1976. Revisão taxonômica do gênero Peltogyne Vogel (Leguminosae-Caesalpiniodeae). INPA, Manaus-AM, Acta Amazonica v. 6 (1) Supl.. 61p. 
SILVA, M. F.; LISBOA, P. L. B.; LISBOA, R. C. L. 1977. Nomes vulgares de plantas amazônicas.Belém, INPA. 222 p. il.

LOUREIRO, A. A.; SILVA, M. F.; ALENCAR, J. C. 1979. Essências madeireiras da Amazônia. $1^{\mathrm{a}}$. ed. Manaus. Amazonas. SUFRAMA. 2 vol. 432 p.

TOKARNIA, C. H.; DÖBEREINER, J.; SILVA, M. F. 1979. Plantas tóxicas da Amazônia a bovinos e outros herbívoros. Instituto Nacional de Pesquisas da Amazônia. Manaus, Amazonas. 95pp.il.

TOKARNIA, C. H.; DÖBEREINER, J.; SILVA, M. F.(orgs.). 1983. Plantas que matam bovinos na Amazônia. 14 ed. vol.3.

SILVA, M. F. 1986. Dimorphandra (Caesalpiniaceae). Flora Neotropica. New York: New York Botanical Garden. vol.44. 126pp.il.

NOGUEIRA, E.; MELHEM, T. S.; FERNANDES, A.; TEIXEIRA, A. R.;FELIPE, G.M.; SILVA, M. F. 1987. A Botânica no Brasil. Descrição do quadro atual. Linhas de ação. MCT/CNPQ, Brasília. 54pp.

SILVA, M. F. (ed.). 1990. Anais do XXXV Congresso Nacional de Botânica, Manaus 1994. Sociedade Botânica do Brasil / IBAMA, Brasília. 446pp.

CARREIRA, L. M. M.; SILVA, M. F.; LOPES, J.R.C.; NASCIMENTO, L. A. S. 1996. Catálogo de pólen das Leguminosas da Amazônia Brasileira. $1^{\mathrm{a}}$. ed. $137 \mathrm{pp}$.

SILVA, M. F.; SOUZA, L. A. G. de; CARREIRA, L. M. M. 2004. Nomes populares das Leguminosas do Brasil. Manaus, EDUA/ INPA/FAPEAM. (Série : Biblioteca Científica da Amazônia). 236pp.il.

\section{CAPÍTULOS DE LIVROS PUBLICADOS POR MARLENE FREITAS DA SILVA}

NODA, H.; SOUZA, L. A. G.; FONSECA, O. J. M.; SILVA, M. F. 1966. Associações Rizóbios-Leguminosas na Amazônia.. In: NODA, H.; SOUZA, L.A.G; FONSECA, O.J.M. \& SILVA, M.F.. (Org.). 20 anos de contribuições do INPA à pesquisa agronômica no trópico úmido. Instituto Nacional de Pesquisas da Amazônia, Manaus, Amazonas. p. 102-115.

SOUZA, L. A. G.; SILVA, M. F. Associações Rizóbios-Leguminosae na Amazônia.1977. In: NODA, H; SOUZA, L. A. G.; FONSECA, O. J. M. (Org.). 20 anos de contribuições do INPA à pesquisa Agronômica no trópico ùmido. Instituto Nacional de Pesquisas da Amazônia, Manaus. Amazonas. vol. único.

SILVA, M. F..; TAVARES, A. S.; BERRY, P. E. Peltogyne Vogel. Flora of Venezuelan Guayana. Caesalpiniaceae-Ericaceae. In: STEYERMARK, J. A.; BERRY, P. E.; HOLST, B. K.. (Orgs.). Flora of Venezuelan Guayana. Caesalpiniaceae - Ericaceae. $1^{\mathrm{a}}$ ed. St. Louis - Missouri. USA, 1998, v. 4, p. 93-97.

SILVA, M. F.; TAVARES, A. S. Dimorphandra Schott. Caesalpiniaceae-Ericaceae.. In: STEYERMARK, J. A.; BERRY, P. E.; HOLST, B. K.. (Orgs.). Flora of Venezuelan Guayana. Caesalpiniaceae - Ericaceae. 1 ed. St. Louis, 1998, v. 4, p. 58-62.
SILVA, M. F.; TAVARES, A. S. ; BERRY, P. E. . Cynometra L. Flora of Venezuelam Guayana. Caeasalpiniaceae - Ericaceae. . In: STEYERMARK, J. A.; BERRY, P. E.; HOLST, B. K.. (Org.). Flora of the Venezuelan Guayana.. 1 ed. St.Louis, 1998, v. 4, p. 49-51.

SOUZA, L. A. G.; SILVA, M. F.. As leguminosas do Arquipélago das Anavilhanas. In: FERREIRA, E.G. (Orgs.). O Arquipélago das Anavilbanas. 1 ed. Manaus, 2000, v. 1, p. -

SOUZA, L. A. G.; SILVA, M. F. Bioeconomical potential of Legumonosae from the Lower Negro River, Amazon, Brazil.. In: BUSSMANN, R. W. \& LANGE, S. (Orgs.). Conservación de la Biodiversidad en los Andes y la Amazonía. Cusco, 2001, v. Único, p. 521-537.

SOUZA, L. A. G.; TEIXEIRA, L. M.; SILVA, M. F. Efeito da inoculação de mudas de jaranduba (Macrosamanea pubiramea - Leg.Mim.) com rizóbios, em dois solos da Amazônia.. In: LISBOA, P. L. B.. (Org.). Caxiuanã: populações, meio físico e diversidade biológica. 1 ed. Belém, PA, 2002, v. único, p. 627-639.

\section{ARTIGOS PUBLICADOS POR MARLENE FREITAS DA SILVA EM PERIÓDICOS:}

SILVA, M. F. 1968. Estudos sobre Caryocaraceae - I. Contribuição para o conhecimento da morfologia foliar de Caryocar glabrum (Aubl.) Pers. e C. microcarpum Ducke da Amazônia. Boletim do INPA. Manaus, 28: 3-18.

RODRIGUES, W. A.; SILVA, M. F. 1971. Uma nova Rauia (Rutaceae) na Amazônia. Acta Amazonica, Belém, Pará. 1 (1): 21.

SILVA, M. F. 1968. Estudos sobre Caryocaraceae - II. Contribuição para o conhecimento da morfologia foliar de Caryocar villosum (Aubl.) Pers. e C. pallidum A. C. Smith da Amazônia. Boletim do INPA. Manaus, 29: 1-15.

SILVA, M. F. 1971. Os tipos do Herbário do INPA-I. Acta Amazonica. Belém, Pará, Suplemento, I:19-32.

SILVA, M. F.; RODRIGUES, W. A. 1971. Novas espécies da flora amazônica. Acta Amazonica, Belém, Pará, 1(2): 33-34.

SILVA, M. F. 1971. Styracaceae da Amazônia. Acta Amazonica. 1 (2):23

SILVA, M. F. 1972. Uma nova Styrax (Styracaceae) na Amazônia. Acta Amazonica, Belém, Pará, 2 (1):17-19.

SILVA, M. F.; LOUREIRO, A. A. 1972. Contribuição ao estudo dendrológico de cinco Parkia (Leguminosae) da Amazônia. Acta Amazonica, 2 (2):71-84.

SILVA, M. F.; LOUREIRO, A. A. 1973. Contribuição ao estudo dendrológico de cinco Leguminosas da Amazônia. Acta Amazonica, 3 (2):7-31.

PRANCE, G. T.; SILVA, M. F. 1975. Revisão taxonômica das espécies amazônicas de Rhizophoraceae. Acta Amazonica, Belém, Pará, 5 (1):5-22.

SILVA, M. F. 1976. Insetos que visitam Theobroma grandiflorum (Willd. ex Spreng.) Schum. (Sterculiaceae). Acta Amazonica, 6(1): 49-54. 
PRANCE, G. T.; RODRIGUES, W. A.; SILVA, M. F. 1976. Inventário florestal de um hectare de mata de terra firme no $\mathrm{km} 3 \mathrm{da}$ estrada Manaus-Itacoatiara. Acta Amazonica, Belém, Pará, 6 (1):9-35.

SILVA, M. F. 1976. Revisão taxonômica do gênero Peltogyne Vog. (Leguminosae)-Caesalpinioideae). Taxon, U.S.A., 25 (5-6): 651-658.

SILVA, M. F.; LOUREIRO, A. A. 1977. Contribuição ao estudo dendrológico de 3 espécies de Qualea (Vochysiaceae) da Amazônia. Acta Amazonica, 7 (3):407-416.

SILVA, M. F. ; FORERO, E. 1977. Instrucciones para coleccionar plantas. Notas Divulgativas, Bogotá. Colômbia, 8: 1-18.

SILVA, M. F. 1978. Notas adicionais à Heliconia tarumaensis Barreiros. Acta Amazonica, Belém, Pará, 8 (4): $591-593$.

SILVA, M. F..; DIAS, A. P. 1978. Algumas plantas cianogenéticas da região amazônica. Acta Amazonica, Belém.Pará, 8 (4): 679-686.

SILVA, M. F.; GRAHAM, A. 1980. Unique pollen types in the Caesalpinioideae (Leguminosae).Grana, Kent State University. USA, 19:79-84.

SILVA, M. F.; GRAHAM, A. 1981. Jacqueshuberia Ducke (Leg. Caesalp.) um gênero exclusivamente neotropical.. Acta Amazonica, 10 (4): 747-754.

SILVA, M. F.1981. Novas espécies de Dimorphandra Schott (Leguminosae-Caesalpinioideae) da região tropical. Acta Amazonica, Belém.Pará, 11 (1): 53-59.

LOUREIRO, A. A.; SILVA, M. F.1981. Estudo dendrológico e anatômico do lenho de 7 espécies e 3 subespécies de Dimorphandra (Leg,Caesalp.). Acta Amazonica, 11 (3): 561583.

RODRIGUES, W. A.; SILVA, M. F.1981. O herbário do INPA. Acta Amazonica,11(4): 667-669.

RODRIGUES, W. A.; SILVA, M. F.; SILVA, A. S. ; RIBEIRO, M. N. G.1981. Criação e evolução histórica do INPA. Acta Amazonica, Belém. Pará, 11 (1):7-23.

TOKARNIA, C. H.; DÖBEREINER, J.; SILVA, M. F.1981. Intoxicação por Palicourea grandiflora (Rubiaceae) em bovinos no Território de Rondônia. Pesquisa Agropecuária Brasileira, Rio de Janeiro. RJ, 1(3): 85-94.

BALICK, M.; ANDERSON, A.; SILVA, M. F.1982. Palm Taxonomy in Brazilian Amazonian: the state of systematic collections in regional herbaria. Brittonia, New York.NY, 34 (4): 463477.

DOBEREINER, J.; TOKARNIA, C. H.; SILVA, M. F. 1983. Intoxicação por Arrabidaea bilabiata (Bignoniaceae) em bovinos na região amazônica do Brasil. Pesquisa Veterinária Brasileira, 3 (1):17-24.

BRANCH, L.; SILVA, M. F.1983. Folk medicine of Alter do Chão. Pará. Brasil. Acta Amazonica, 13 (5-6): 737-797.

ROCHA, A. I.; LUZ, A. I. R.; SILVA, M. F.1984. A presença de alcalóides em espécies botânicas da Amazônia. Menispermaceae. Acta Amazonica 14 (1-2): 244-246..
SILVA, M. F.; MESQUITA, A. L. 1984. Enterolobium barnebianum A. L. Mesquita et M.F.Silva uma nova Mimosaceae para a Amazônia Brasileira. Acta Amazonica, 14 (1-2): 153-158.

PRANCE, G. T.; NELSON, B. W.; SILVA, M. F.; DALY, D. C. 1984. Projeto Flora Amazônica : eight years of binational botanical expeditions. Acta Amazonica, 14 (1-2): 5-29.

LOUREIRO, A. A.; SILVA, M. F.; VASCONCELOS, F. J. 1984. Contribuição ao estudo anatômico do lenho de sete espécies de Dimorphandra (LeguminosaeCaesalpinioideae). Acta Amazonica, 14 (1-2): 289-313.

SILVA, M. F.; MIRANDA, I. P. A.; BARBOSA, E. M. 1986. Aspectos sobre a polinização do dendezeiro Elaeis guianensis e do caiaué E .oleifera. Acta Amazonica, 16-17 (n. Único): 209218.

MOREIRA, F. M. S.; SILVA, M. F.1987. Associações Rizobium Leguminosas no Estado de Rondônia. Acta Amazonica, 16-17 (n. Único/Supl): 7-17.

SILVA, M. F.; GOLDMAN, G. H.; MOREIRA, F. M. S.; MOREIRA, F. W. 1988 . Germinação natural de 10 leguminosas arbóreas da Amazônia - I. Acta Amazonica, 18 (1-2): 9-26.

SOUZA, L. A. G.; SILVA, M. F.; MOREIRA, F. W.1988. Levantamento da capacidade de nodulação e/ou fixação de nitrogênio em Leguminosas nativas da Amazônia. Pesquisa Agropecuária Brasileira.

NELSON, B. W.; SILVA, M. F.; FERREIRA, C. A. C. 1989. How reliable database for interpreting Quaternary biogeography and Paleoclimate of Brazilian Amazon. Associação Brasileira de Estudos Quaternários, 1 (n. Especial): 92-98.

SILVA, M. F.; CARREIRA, L. M. M.; TAVARES, A. S.; RIBEIRO, I.; JARDIM, M. A. G.; ALBUQUERQUE, M. G. L.; OLIVEIRA, J.1989. Leguminosas da Amazônia Brasileira. Lista Prévia. Acta Botanica Brasilica, 2 (1): 193-237..

NELSON, B. W.; FERREIRA, C. A. C.; SILVA, M. F.; KAWASAKI, M. L.1990. Endemism Center Refugia and Botanical colections density in Brazilian Amazon. Nature, New York.NY, 345 (6277): 714-716.

SILVA, M. N. P.; AUGUSTO, N. F.; SILVA, M. F.; SILVA, M. B. C. E. 1991. Produção de Docentes e de Discentes da UTAM - I.. UTAM. Publicações Avulsas, Manaus.Amazonas,vol. único:115.

MOREIRA, F. M. S.; SILVA, M. F.; FARIA, S. M.1992. The occurrence of nodulation in legumes species in the Amazon region of Brazil. New Phytologist 121: 563-570.

RANKIN-DE-MERONA, J. ; PRANCE, G. T. ; HUTCHINGS, H. R. W. ; SILVA, M. F.; RODRIGUES, W. A.; UELING, M. E. 1992b. Preliminary results of a large scale tree inventory of rain forest in Central Amazon. Acta Amazonica, 22 (4): 493-534.

TAVARES, A. S.; SILVA, M. F. 1992. Distribuição geográfica do gênero Cynometra L. (Caesalpiniaceae) no mundo. Acta Amazonica, 22 (1): 17-22.

SILVA, M. F. ; CARREIRA, L. M. M.; CORTÊS, A. M. L.1993. Leguminosas da Amazônia Brasileira - II. Cedrelinga Ducke (Leg. Mimos.). Bol. Museu Paraense Emílio Goeldi, Sér. Botânica, 8 (1): 143-156. 


\section{ACTA \\ AMAZONICA}

MOREIRA, F. M. S.; SILVA, M. F. 1993. Associação Rizóbio Leguminosas na Amazônia. Bol. Museu Paraense Emílio Goeldi, Sér. Botânica, 9 (2):129-141.

MOREIRA, F. M. S.; MAGALHAES, L. M. S.; SILVA, M. F. ; MOREIRA, F. W.; SCARCELlO, J. A. 1993. Associação Rizóbio Leguminosas na Amazônia - II. Bol. Museu Paraense Emílio Goeldi, Sér. Botânica, 9 (2): 267-277.

SOUZA, L. A. G.; SILVA, M. F.; MOREIRA, F. W.1994. Capacidade de nodulação de 100 leguminosas da Amazônia.. Acta Amazonica, Belém, Pará 24 (1)-2, p. 9-18.

SILVA, M. F.; CARREIRA, L. M. M. 1994 . Leguminosas da Amazônia Brasileira- III. Jacqueshuberia Ducke (Leg.Caesalp.). Bol. Museu Paraense Emílio Goeldi, Sér. Botânica,10 (2): 183 -188.

SILVA, M. F.; CARREIRA, L.M. M.1994. Leguminosas da Amazônia Brasileira - IV. Heterostemon Desf. (Leg. Caesalp.). Bol. Museu Paraense Emílio Goeldi, Sér. Botânica, Belém, Pará, 10 (2): 189-220.

MOREIRA, F. M. S.; SILVA, M. F.; MOREIRA, F. W.; SOUZA, L. A. G. 1994. Associação Rizóbio - Leguminosas na Amazônia - III. Bol. Museu Paraense Emílio Goeldi, Sér. Botânica, 10 (2): 295-302.

RIBEIRO, J. E. L. S.; NELSON, B. W.; SILVA, M. F.; MARTINS, L. S. S.; HOPKINS, M. J. G. 1994. Reserva Florestal Ducke. Diversidade e Composição da Flora Vascular. Acta Amazonica, Manaus, Amazonas, 24 (1-2): 19-30.

MOREIRA, F. W.; MOREIRA, F. M. S.; SILVA, M. F.. 1995. Germinação, crescimento inicial e nodulação em viveiro da saboarana Swartzia laevicarpa Amshoff.. Acta Amazonica, 25 (3/4): 149-160.

SILVA, M. F.; CORRÊA, Y. M. B. 1995. Plantas ruderais de Manaus e seu potencial de utilização. Bol. Museu Paraense Emílio Goeldi, Sér. Botânica, Belém, Pará. 11 (2): 239-254.

SOUZA, L. A. G. ; SILVA, M. F..1998. Tratamentos escarificadores em sementes duras de sete leguminosas nativas da Ilha de Maracá. Roraima.Brasil. Bol. Museu Pararense Emílio Goeldi, Sér. Botânica, Belém, Pará, 14 (1): 11-32.

SOUZA, L. A. G.; DANTAS, A. R. ; MATOS, R. B. ; SILVA, M. F. ; SAMPAIO, P. T. B. 2000 . Período de frutificação e viabilidade das sementes do acapu Vouacapoua americana Aubl. Leg. Caesalp. do Médio Rio Tocantins. Pará. Brasil. Bol. Museu Paraense Emílio Goeldi, Sér. Botânica, Belém, Pará, 16 (1): 3-21.

MARTINS, S. L.; SOUZA, L. A. G. ; SILVA, M. F. 2000. Efeitos de tratamentos pré-germinativos na superação da dormência das sementes de oito espécies de leguminosas herbáceas. Universidade do Amazonas, Manaus.

SILVA, M. F.; BARBOSA, A. P. ; SOUZA, L. A. G. 2000. Potencial bioeconômico das leguminosas do baixo rio Negro. Amazonas. Revista Parcerias Estratégicas, Brasília.

SILVA, M. F.; ALBERTINO, S. M. F.; SOUZA, L. A. G.; SILVA, J. F..2000. Superação da dormência de sementes de oito espécies da Leguminosas herbáceas submetidas a tratamentos pré-germinativos. Ciências Agrárias, Manaus,.Amazonas, 9 (1-2): 1-23..

SILVA, J. A. C. ; SILVA, M. F..2001 . Uma nova Sterculiaceae para a Reserva Ducke, Manaus, Amazonas. Acta Amazonica, 31 (3): 375-380.

FALCÃO, M. A. ; PARALUPPI, N. D.; CLEMENT, C. R.; E.KERR, W.; SILVA, M. F.2001. Fenologia e produtividade do abacate (Persea americana Mill.) na Amazônia Central. Acta Amazonica, 31 (1): 3-9.

SILVA, M. F.; ATAYDE, C. M.; DÜNISCH, O. 2001. Wood anatomical technological properties of tree species of Hymenaea $\mathrm{L}$. (jatobá) on plantation and naturally grown trees. Annal of Vermont Brazilian Workshop On Neotropical Ecosystem.

SOUZA, L. A. G. ; SILVA, M. F.; DANTAS, A. R. 2001. Germinação das sementes e inoculação de mudas de macacaúba, Platymiscium duckei Huber (LEG. PAP.) com rizóbios, em latossolo amarelo (Oxisol). Acta Amazonica, 31(4): 547 556.

SOUZA, L. A. G. DE; SILVA, M. F.. 2002. Bioeconomical potential of Leguminosae from the lower Negro River, Amazon, Brazil. Proceedings of the International Congress of Biodiversity. Cusco, Peru. Vol. único: 520-538.

SILVA, M. F.; SOUZA, L. A. G. .2002. Levantamento das Leguminosas do Arquipélago das Anavilhanas. Baixo Rio Negro. Amazonas. Bol Museu Paraense Emílio Goeldi Sér Bot, Belém.Pará,18 (1): 03-36.

BARATA, F. C. A.; CARREIRA.L.M.M.; SILVA, M. F. (no prelo). Leguminosas da Amazônia brasileira - IX. O pólen do gênero Diptychandra Tul. (Leg. Caesalp.). Bol. Museu Paraense Emílio Goeldi, série Ciências Naturais, Belém, Pará. 1 (2).

SILVA, M. F.; CARREIRA, L.M.M.; SOUZA, L. A. G DE. (no prelo). Leguminosas da Amazônia Brasileira - X. Martiodendron Gleason (Leguminosae, Caesalpinioideae). Bol. Museu Paraense Emílio Goeldi, série Ciências Naturais, Belém, Pará, 1 (2).

GURGEL, E. S. C.; CARVALHO, A. C. M.; SANTOS, J. U. M. DOS; SILVA, M. F. (no prelo). Virola surinamensis (Rol. ex Rottb.) Warb. (Myristicaceae). Aspectos morfológicos do fruto, semente, germinação e plântula. Bol. Museu Paraense Emílio Goeldi, série Ciências Naturais, Belém, Pará.

GURGEL, E. S. C.; SILVA, M. F.; CARVALHO, A. C. M.; SANTOS, J. U. M. DOS. (no prelo). Jacaranda copaia (Aubl.) D. Don. subsp. spectabilis (Mart. ex A. DC.) Gentry: Aspectos morfológicos do fruto, semente, germinação e da plântula. Bol. Museu Paraense Emílio Goeldi, série Ciências Naturais, Belém, Pará.

GURGEL, E. S. C.; SILVA, M. F.; CARREIRA, L. M. M.; SANTOS, J. U. M. DOS. (no prelo). Morfologia do fruto e da semente de três espécies de Senna (Bauhin) Miller (Leguminosae Caesalpinioideae). Revista Brasileira de Botânica, São Paulo - SP. 
